\title{
Richtlinien «Patientenverfügung»: Kurzfassung und Musterfragen zur Werthaltung
}

Schweizerische Akademie der Medizinischen Wissenschaften (SAMW)

\footnotetext{
* Die entsprechenden Texte betreffen immer beide Geschlechter der genannten Personengruppen.
}

Die «Alltagstauglichkeit» von Richtlinien ist ein wichtiger Faktor für deren Verwendung in der Praxis; auf diesen Umstand weisen sowohl die Stellungnahmen im Rahmen der Vernehmlassung zu einzelnen Richtlinien der Schweizerischen Akademie der Medizinischen Wissenschaften (SAMW) als auch die Antworten einer Evaluationsstudie hin. Dabei spielt selbstverständlich auch der Umfang einer Richtlinie eine Rolle; dieser ist je nach Thema der Richtlinie unterschiedlich gross. Insgesamt zeichnet sich in den letzten Jahren aber eine deutliche Tendenz zu umfangreicheren Richtlinien ab. Bei der Evaluationsstudie hatte die Hälfte der Antwortenden angegeben, dass sie die SAMW-Richtlinien wichtig fänden, aber zu wenig Zeit hätten, sich damit auseinanderzusetzen. Abhilfe könnten Kurzfassungen der Richtlinien bieten, die einen raschen Überblick über die zentralen Punkte geben. Die SAMW hat dies zum Anlass genommen, erstmals eine solche Kurzfassung zu veröffentlichen, und zwar zu den Richtlinien «Patientenverfügung»; gleichzeitig hat sie auch «Musterfragen zur Werthaltung» zusammengestellt. Die Kurzfassung ersetzt die Lektüre der Richtlinien nicht, sondern soll Hinweise für die Praxis vermitteln. Die Kapitelverweise beziehen sich auf die Richtlinien.

\section{Wegleitung Patientenverfügung}

Eine Patientenverfügung hält den Willen eines Patienten* für den Fall einer Urteilsunfähigkeit im Voraus fest; sie kann sich auf verschiedene Anwendungsbereiche beziehen. Den Ärzten, Pflegefachpersonen und weiteren Fachpersonen kommen dabei vielfältige Aufgaben zu: Sie geben grundlegende Informationen zur Patientenverfügung, sprechen das Thema in geeigneten Situationen an, informieren über die inhaltlichen und formalen Anforderungen der Patientenverfügung, sind beim Erstellen behilflich oder überprüfen mit dem Patienten eine bereits erstellte Patientenverfügung auf ihre Aktualität. Es ist wichtig, dass das Gespräch über die Patientenverfügung in einer dem Patienten angepassten Form erfolgt. Ängste vor bestimmten Erkrankungen oder Massnahmen, negative Erfahrungen mit bestimmten Behandlungsformen oder Befürchtungen sollen thematisiert werden.

Ist ein Patient nicht mehr urteilsfähig, hat das Behandlungs- und Betreuungsteam die Aufgabe, eine vorliegende Patientenverfügung in einer konkreten Behandlungssituation umzusetzen, sofern die Patientenverfügung dies vorsieht.

Nicht alle Ärzte und Pflegefachpersonen müssen «Spezialisten» bezüglich der Patientenverfügung sein.
In grösseren Institutionen ist es sinnvoll, wenn es Mitarbeitende gibt, die über die notwendigen Kenntnisse verfügen und so Fragen von Fachpersonen oder Patienten beantworten und gegebenenfalls auch Beratung anbieten können. Zudem gibt es Organisationen, die Ärzte, Pflegefachpersonen und Verfügende beim Erstellen unterstützen.

\section{Ist die Patientenverfügung verbindlich? (Kapitel 2 und 3)}

Gesetzliche Regelungen zur Patientenverfügung bestehen bislang erst auf kantonaler Ebene, jedoch nicht in allen Kantonen. Mit dem Inkrafttreten des neuen Erwachsenenschutzgesetzes (frühester Zeitpunkt 2012) wird die Patientenverfügung im eidgenössischen Recht explizit verankert sein. Danach muss eine Patientenverfügung befolgt werden, ausser sie verstösst gegen gesetzliche Vorschriften oder es gibt Anhaltspunkte, dass der Patient die Meinung geändert hat. Bereits heute gilt: Je klarer eine Patientenverfügung ist, desto gewichtiger ist sie im Entscheidungsprozess.

\section{Wer kann eine Patientenverfügung erstellen und was ist zu beachten?}

(Kapitel 3.2 bis 3.4 und 5.2.2)

Jede Person, die urteilsfähig ist, kann eine Patientenverfügung erstellen. Das gilt auch für urteilsfähige Jugendliche. Der Entschluss zum Verfassen einer Patientenverfügung muss auf freiem Willen beruhen, d.h. niemand darf zum Verfassen einer Patientenverfügung gedrängt werden. Eine Patientenverfügung muss schriftlich erstellt, datiert und handschriftlich unterzeichnet sein. Sie kann jederzeit geändert bzw. schriftlich oder mündlich aufgehoben werden. Der Patient sollte über die Wichtigkeit der Aktualisierung der Patientenverfügung informiert werden (vgl. oben). Es wird deshalb empfohlen, die Patientenverfügung regelmässig zu überprüfen. Hat sich die persönliche Einstellung gegenüber Leben, Krankheit und Sterben («persönliche Werthaltung») oder die gesundheitliche Situation geändert, sollte die Patientenverfügung angepasst werden.

\section{Ist jede Patientenverfügung gültig? \\ (Kapitel 3.4 und 9)}

Grundsätzlich müssen die formalen Anforderungen erfüllt sein: Schriftlichkeit, Urteilsfähigkeit, Freiwilligkeit, Datierung, eigenhändige Unterschrift.

Die Gültigkeit einer Patientenverfügung ist nicht befristet. Wichtig ist, dass eine Patientenverfügung den aktuellen Willen des Patienten wiedergibt. Solange es 
Inhalte der Patientenverfügung (Kapitel 4)

- Angaben zur Identität des/der Verfügenden (Name, Vorname, Geburtsdatum)

- Bestätigung der Urteilsfähigkeit («Im Besitz meiner geistigen Kräfte und nach reiflicher Überlegung verfüge ich hiermit für Situationen, in denen ich krankheits- oder unfallbedingt nicht fähig bin, meinen aktuellen Willen zu äussern, Folgendes»). Anmerkung: In Situationen, bei denen später die Urteilsfähigkeit angezweifelt werden könnte (z.B. beginnende Demenz-Erkrankung, psychiatrisches Leiden), kann zur Vermeidung von Unsicherheiten die Urteilsfähigkeit zusätzlich durch einen Arzt/eine Drittperson bestätigt werden.

- Beschreibung der persönlichen Werthaltung: Was bedeutet Lebensqualität und «Sterben in Würde» konkret für den Verfügenden? Welche persönlichen Überzeugungen, Ängste, Erwartungen sind bei medizinischen Entscheidungen in Grenzsituationen zu beachten? Vgl. «Musterfragen» weiter unten.

- Bezeichnung mindestens einer Vertretungsperson und Kontaktangaben zu dieser Person. Idealerweise wird auch eine Ersatzperson genannt.

- Angaben, für welche Situationen die Patientenverfügung erstellt wird bzw. in welchen Situationen sie zur Anwendung kommen soll.

- Angaben zu den Zielen einer Behandlung in bestimmten Situationen

- Einwilligung in bzw. Ablehnung von spezifischen medizinischen Massnahmen: Im Fall einer bereits bekannten Erkrankung zum Zeitpunkt des Erstellens der Patientenverfügung sollte diese erwähnt werden und die $\mathrm{Pa}$ tientenverfügung auf die Erkrankung und deren wahrscheinlichen Verlauf, mögliche Komplikationen und Massnahmen abgestimmt werden.

- Bereitschaft zur Organspende

- Umgang mit dem Körper nach dem Tod (Autopsie)

- Datum und Unterschrift

keine Anhaltspunkte (Hinweise der Vertrauensperson oder der Angehörigen, Hinweise von Ärzten, Pflegenden und Betreuenden) gibt, dass der Patient in der Zwischenzeit seine Meinung geändert hat, kann davon ausgegangen werden, dass die Patientenverfügung dem aktuellen Patientenwillen entspricht. Gibt das non-verbale Verhalten des Patienten (z. B. bei Demenzpatienten) Anlass zur Annahme, dass die Patientenverfügung nicht dem aktuellen Willen entspricht, muss dies unter Einbezug der Vertretungsperson und An- gehörigen sorgfältig abgeklärt werden. Der Entscheid sollte einvernehmlich im Behandlungs- und Betreuungsteam gemeinsam mit allfälligen Vertretungspersonen und Angehörigen getroffen werden. Die Abweichung vom Wortlaut in der Patientenverfügung muss im Patientendossier festgehalten und begründet werden (vgl. Kapitel 9 «Willensänderung»).

\section{Wann kommt eine Patientenverfügung} zur Anwendung und wie wird vorgegangen? (Kapitel 8)

Eine Patientenverfügung kommt nur zur Anwendung, wenn ein Patient urteilsunfähig ist, d.h. sich nicht mehr selbst äussern kann. Ist der Patient hingegen urteilsfähig, gilt der aktuell geäusserte Wille.

Ist ein Patient urteilsunfähig, müssen folgende Abklärungen getroffen werden:

- Liegt eine Patientenverfügung vor (Suche nach einem Ausweis, Befragung Hausarzt oder Angehörige)?

- Hat der Patient eine Vertretungsperson bezeichnet? Falls ja, muss diese informiert und in die Behandlungsplanung einbezogen werden.

Der Behandlungsentscheid aufgrund der Patientenverfügung sollte einvernehmlich zwischen Behandlungsteam, Vertretungspersonen und Angehörigen getroffen werden.

Muss die Patientenverfügung auch in der Notfallsituation umgesetzt werden? (Kapitel 8) Die Dringlichkeit, mit der in einer Notfallsituation Massnahmen eingeleitet werden müssen, z. B. bei einem Unfall auf der Strasse, lassen es in der Regel nicht zu, vorgängig das Vorhandensein einer Patientenverfügung abzuklären. Die nötigen lebenserhaltenden Massnahmen müssen deshalb eingeleitet werden. Es muss aber danach geprüft werden, ob eine Patientenverfügung verfasst wurde, diese muss bei der Behandlungsplanung einbezogen und eingeleitete Massnahmen müssen unter Umständen abgebrochen werden.

Wie weiss man, dass eine Patientenverfügung erstellt wurde? (Kapitel 6)

Es ist Sache des Verfügenden, über das Vorhandensein einer Patientenverfügung zu informieren. Dies kann mündlich, z. B. beim Spitaleintritt, geschehen. Es kann auch in der Form eines Ausweises oder in Zukunft durch den Eintrag auf der Versichertenkarte informiert werden. Der behandelnde Arzt sollte jedoch immer auch nachfragen, ob eine Patientenverfügung verfasst wurde.

Patientenverfügungen werden auf sich getragen, dem Hausarzt oder der Vertretungsperson abgegeben oder auf einer Hinterlegungsstelle aufbewahrt.

Hat der Arzt Kenntnis vom Vorhandensein der Patientenverfügung und dem Hinterlegungsort, fordert er die Patientenverfügung an. 
Was ist zu tun, wenn sich Arzt, Vertretungsperson oder Angehörige nicht einig sind? (Kapitel 10) Eine Patientenverfügung muss in eine konkrete Situation übersetzt werden. Es kann vorkommen, dass Vertretungsperson, Behandlungsteam und Angehörige nicht gleicher Meinung sind. Dann sollte eine Ethikberatung zu Hilfe gezogen werden. Führt dies zu keiner Einigung, muss die gesetzlich vorgeschriebene Stelle (Vormundschaftsbehörde, ab Inkrafttreten des neuen Erwachsenenschutzrechts: Erwachsenenschutzbehörde) beigezogen werden.

\section{Musterfragen zur Werthaltung}

Die folgenden Fragen zu den jeweiligen Themenkreisen wurden als Hilfestellung erarbeitet, um die Wertvorstellungen des Patienten zu dokumentieren («Werteanamnese»). Sie können im Beratungsgespräch eingesetzt oder vom Verfügenden direkt beantwortet werden. Wichtig ist, dass die Fragen aus der aktuellen Lebenssituation beantwortet werden. Die Werteanamnese ist demnach immer eine Momentaufnahme, die sich im Lauf der Zeit verändern kann und deshalb allenfalls angepasst werden muss.

Die Beschreibung der persönlichen Wertvorstellungen ist in einer Patientenverfügung von grosser Bedeutung. Sie gibt Aufschluss, welche Lebenseinstellungen, Befürchtungen, Werte und Erwartungen für den Patienten bestimmend sind. Die Informationen zur Werthaltung dienen auch als Orientierung in Situationen, wenn eine Patientenverfügung keine genauen Angaben zu einzelnen medizinischen Massnahmen macht. (Kapitel 4.1.)

\section{Motivation}

Was bewegt Sie, eine Patientenverfügung zu verfassen? Gibt es einen konkreten Anlass? Was wollen Sie mit einer Patientenverfügung erreichen bzw. was wollen Sie damit vermeiden? Haben Sie mit Ihren Angehörigen darüber gesprochen? Haben Sie mit Ihrem Hausarzt darüber gesprochen?

\section{Das Leben zwischen Geburt und Tod}

Wo sehen Sie sich in Ihrem Leben? Wie wichtig ist es für Sie, noch lange zu leben? Wären Sie bereit, für den Gewinn an Lebensjahren mit Einschränkungen (z. B. Pflegebedürftigkeit) zu leben? Oder wollen Sie auf Lebensjahre verzichten und dafür möglichst unabhängig leben? Was heisst für Sie persönlich und konkret «Sterben in Würde»? Was ist die Rolle naher Angehöriger/Ihrer Familie bzw. welche Aufgaben sind sie bereit zu übernehmen und was kann ihnen zugemutet werden?

\section{Lebensqualität}

Was macht Ihr Leben lebenswert? Welche Aktivitäten, welche Inhalte und Werte bestimmen Ihr Leben aktuell? Ist es für Sie mit Blick auf eine mögliche Erkrankung oder das fortschreitende Alter denkbar, dass sich Ihre Vorstellungen über Lebensqualität verändern (z. B. bezüglich Kommunikationsfähigkeit, Mobilität, geistige Verfassung)? Wie wichtig ist Schmerzfreiheit? Wären Sie bereit, dafür ein getrübtes oder im Extremfall ein ausgeschaltetes Bewusstsein in Kauf zu nehmen?

Erfahrungen mit Krankheit, Sterben, Tod Haben Sie bereits persönlich Krankheitserfahrungen gemacht? Oder haben Sie Erfahrungen mit Kranksein über Dritte gemacht (z.B. Eltern, Partner, Freunde)? Wie prägen diese Erfahrungen Ihr Verhältnis zu Medizin, Pflege und zur Inanspruchnahme medizinischer Leistungen? Leben Sie aktuell mit Einschränkungen oder Erkrankungen? Sehen Sie einen Sinn im Leben, auch wenn Sie stark eingeschränkt oder die Persönlichkeit verändert wäre (z.B. Koma, schwere Demenz)? Wären Sie bereit, für das Überleben in einer solchen Situation medizinische Belastungen in Kauf zu nehmen? Welche Schädigungen bzw. Beeinträchtigungen wären für Sie so schwerwiegend, dass Sie nicht mehr weiterleben möchten?

\section{Persönliche und religiöse Überzeugungen}

Haben Sie religiöse, spirituelle oder weltanschauliche Überzeugungen? Gehören Sie einer Kirche oder Gruppierung an? Prägen diese Überzeugungen den Umgang mit Ihrem Leben in «Grenzsituationen», d.h. wenn Ihr Leben gefährdet wäre (Leben erhalten oder sterben lassen). Gibt es Punkte oder Rituale, die aufgrund Ihrer weltanschaulichen oder religiösen Überzeugungen nach dem Tod beachtet werden sollten (Umgang mit dem Körper usw.)? Wären Sie einverstanden mit der Weitergabe Ihrer Organe oder Gewebe nach Ihrem Tod (Organspende)?

Der vollständige Text der Richtlinien und Empfehlungen «Patientenverfügung» ist unter www. samw.ch/de/Ethik/Richtlinien/Aktuell-gueltigeRichtlinien.html online abrufbar und kann beim Generalsekretariat der Schweizerischen Akademie der Medizinischen Wissenschaften (SAMW), Petersplatz 13, 4051 Basel, bestellt werden. 